\title{
Silhouette differences among cats do not suggest a general selection for paedomorphosis
}

\author{
Pere M. Parés-Casanova* \\ Department of Animal Production, University of Lleida, 25198 Lleida, Spain \\ (Received: April 10, 2012; Revised: August 9, 2012; Accepted: December 10, 2012)
}

\begin{abstract}
Paedomorphosis is the retention of juvenile morphology at maturity and is important in generating evolutionary change in domestic species and species in the wild. This preliminary study compared morphological characteristics of seven domestic cat pure breeds and felid species from around the world. An original procedure based on elliptical Fourier (EF) methods was applied to head lateral views of specimens and were assessed in order to analyse head profile. For comparative purposes three domestic kittens of different ages and three species of genus Lynx were also used. EF descriptors, allowed for the quantification of the head profile. Using the Fourier transform, reconstruction of the mean head profile revealed that there was a general shape difference between wild cats, domestic cats and kittens. Results suggested that variability in head profile differentiate quite well between adult cats and kittens, but domestic and wild cats appeared grouped into a similar cluster. The similarity between breeds can thus be attributed more to the general head profile than to flatness, i.e. to the general conformation rather than facial profile. Therefore, no effect of paedomorphism on the studied breeds can be undertaken. The present approach opens interesting ethnological perspectives for the aloidic characterisation for domestic breeds.
\end{abstract}

Keywords : aloidism, elliptic Fourier descriptors, morphology, skull

\section{Introduction}

Paedomorphosis is the retention of ancestrally juvenile traits by adults in descendant taxons [1] thus the adults resemble juvenile stages of the ancestral species [19]. Paedomorphosis is considered an important mechanism for producing evolutionary change [7] and domestic animals have been used as models of evolutionary change since Darwin [6]. Physical evidence for paedomorphosis has been described in fossils and domestic dogs [5, 15] and extant horses [3, 12]. To our knowledge, there are not researches on cats.

Quantitative shape evaluation, as an important analysis in morphological descriptions, includes methods such as contour length assessment, fractal analysis, and use of landmarks or Fourier descriptors, one method of estimation being from digitally processed images [9]. Digital image analysis is one way to compensate for the weakness of the current qualitative evaluation of continuous variation in various characteristics. With recent improvements in computer performance, digital image analysis has been applied to quantitative evaluation in various agricultural and biological research such as disease assessment [13, 16, 17].

The aim of this article is to examine data that will allow us to explore evidence of profile differences among seven breeds of cats in comparison with kittens and with wild feline species using head profile evaluation. If physical indicators of paedomorphism are suggested by this initial investigation, further studies of physical paedomorphism would be conducted in a wider range of contemporary domestic cat breeds. Many breeds of cats appear to have large morphological differences between them, but the purpose of this research has not been to classify them, rather to suggest if paedomorphism - understood as a similarity with kitten profile- is present in domestic cat breeds.

\section{Materials and Methods}

\section{Specimens}

Head lateral views of specimens of seven different pure stud booked breeds (Abyssinian, Angora, Bengali, Cornish, Maine, Siamese, Sphynx) from around the world were assessed for head profile. For comparative purposes three domestic kittens of different ages were used. To produce a range of physical similarities between the breeds, leopard (Panthera pardus) and three species of genus Lynx (Bobcat, Lynx rufus; Eurasian Lynx, Lynx lynx; Iberian Lynx, Lynx pardinus) were also studied. Images were taken from public supplied on-line photographs.

*Corresponding author

Tel: +34-973-70-64-60, Fax: +34-973-70-28-74

E-mail: peremiquelp@prodan.udl.cat 


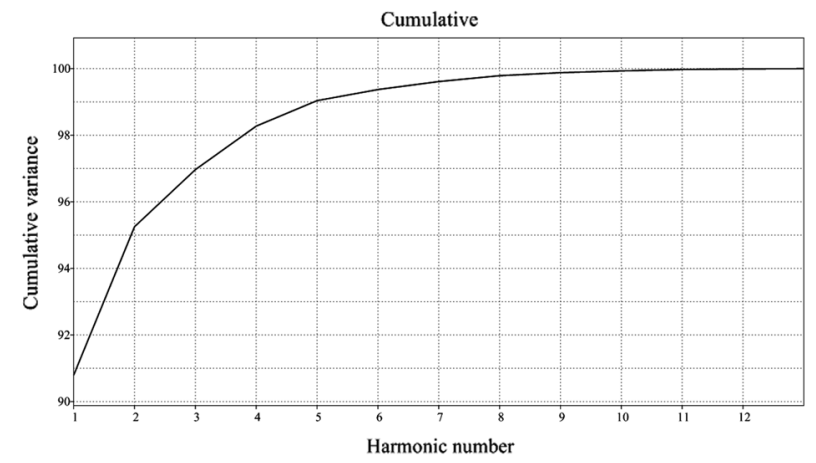

Fig. 1. Cumulative variance (\%) resulting from the Fourier reconstruction of an outline based on increasing numbers of harmonics.

\section{Image processing and contour recording}

From obtained photographs the head contour was manually drawn. Then pictures were then converted to digital images using Epson Stylus DX3800 (Seiko Epson Corporation, Spain) for scanning and produced binary black silhouettes images in BMP format using Photo Suite 4 software (Softonic, Spain). From these images, the closed contours of the heads were extracted and chain coded [4] for the further analysis.

\section{Elliptic Fourier (EF) descriptors and Principal Component Analysis}

EF shape analysis is a technique for generating a set of shape-representative variables from a poor outline suitable for use in statistical comparisons between samples. It has been applied to many organisms. EF decomposition has several advantages over other methods. It does not require the points to be spaced equally. EF coefficients are independent of the outline position on the digitalisation grid. The shape evaluation method is a powerful tool for analysing biological shapes. This method involves complex procedures such as derivation of the descriptors. In general, Fourier expansions of shape data, such as these outlines, are sensitive to information that is usually considered irrelevant, such as location, size and orientation of the object. The coefficients of EF descriptors were calculated by the discrete Fourier transformation of the chain-coded contour through the procedure proposed by Kuhl and Giardina [11] which can remove these sensitivities. Normalised EF descriptors were calculated with Shape software package developed by Iwata and Ukai [10]. This programme provides image analysis, contour recording (shape), derivation of the Fourier descriptors (Chc2Nef) and then principal component analysis on the descriptors. The normalised method used was based on the first harmonic.

The number of harmonics required can also be estimated from the average Fourier power spectrum. The Fourier power of a harmonic is proportional to its amplitude and provides a measure of the amount of shape information described by these harmonics. For the $n$th harmonic, Fourier power $\left(\mathrm{PF}_{\mathrm{n}}\right)$

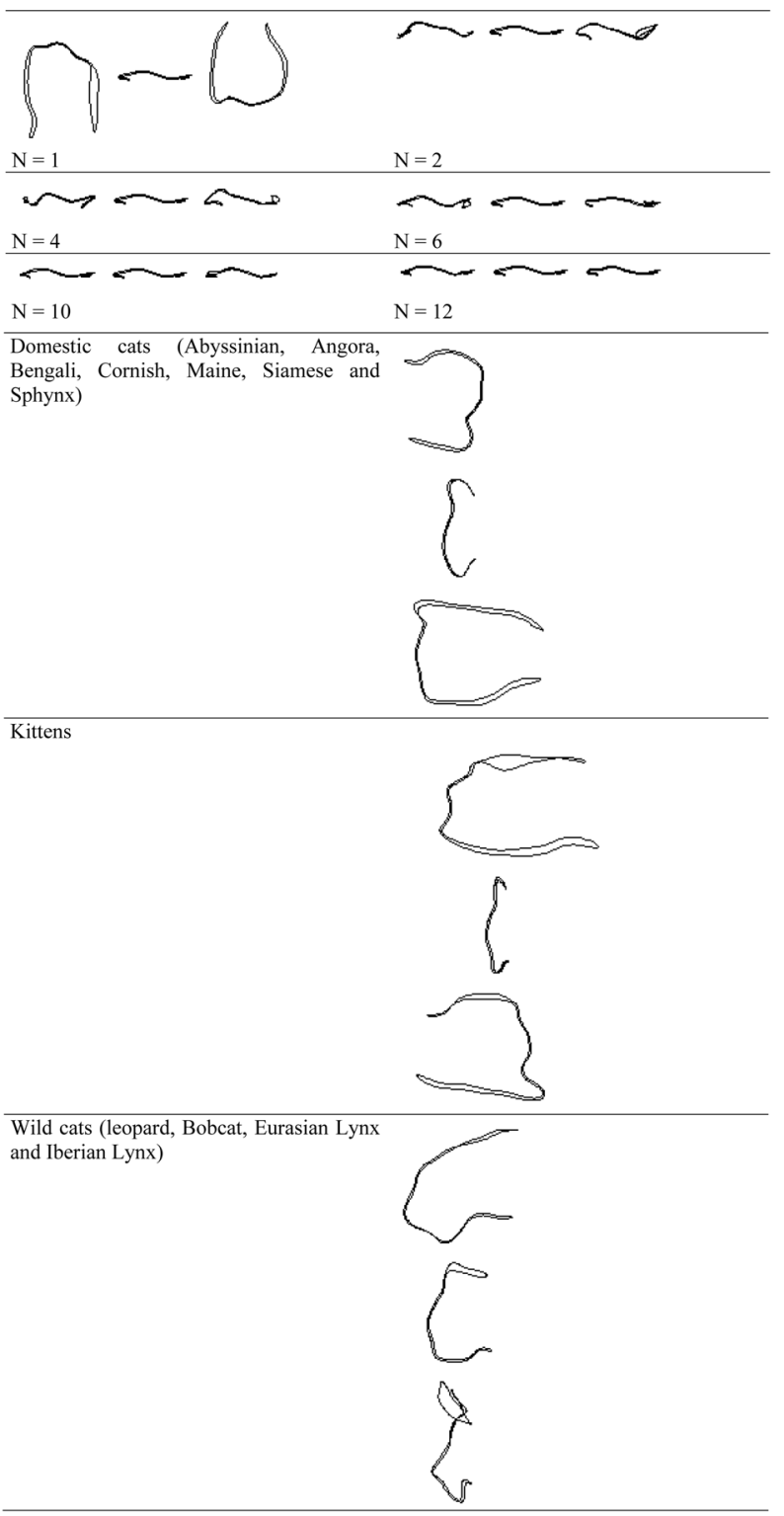

Fig. 2. Shape reproduction (-2StD Mean $+2 \mathrm{StD})$ by addition of Fourier shape components (harmonics).

is given by the expression $\mathrm{PF}_{\mathrm{n}}=\left(\mathrm{A}_{\mathrm{n}}^{2}+\mathrm{B}_{\mathrm{n}}^{2}+\mathrm{C}_{\mathrm{n}}^{2}+\mathrm{D}_{\mathrm{n}}^{2}\right)^{-2}$, where $\mathrm{A}_{\mathrm{n}}, \mathrm{B}_{\mathrm{n}}, \mathrm{C}_{\mathrm{n}}$ and $\mathrm{D}_{\mathrm{n}}$ are the Fourier coefficients of the $n$th harmonic. Then, the calculated power percentage $\left(\mathrm{PF}_{\mathrm{c}}\right)$ was calculated by: $\mathrm{PF}_{\mathrm{c}}=\Sigma^{\mathrm{n}} \mathrm{PF}_{\mathrm{n}}$. As the first 11 harmonics reached $99.96 \%$ of the cumulative power, the Fourier analysis indicated that the head shape could be summarised by 12 harmonics (Fig. 1).

Each of the 12 harmonics considered is defined by four coefficients. Therefore, one sample was described by 48 variables, and was used for the analysis. Lastly, a principal coordinates analysis (PCO) was applied using Fourier descriptors. In order to avoid a decrease of the "horseshoe" effect [18] the similarity/distance values were raised to the power of $c=2$ (the "transformation exponent"). Multivariate statistical anal- 


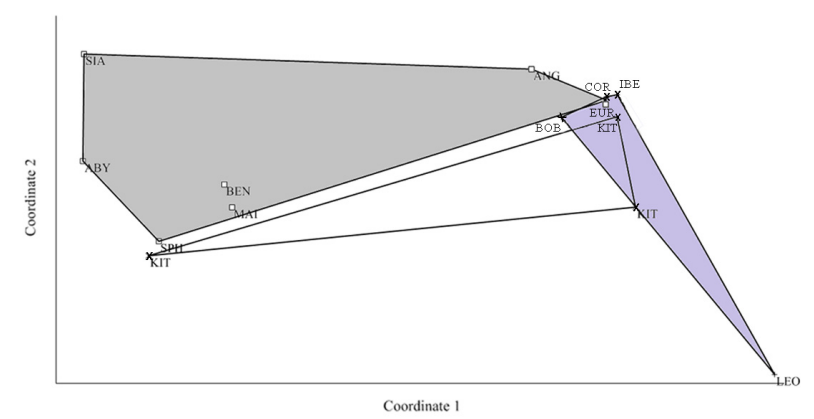

Fig. 3. Breeds plotted in the coordinate system given by the PCO. Average profiles ( $-2 \mathrm{StD}$ Mean $+2 \mathrm{StD}$, from bottom to top) for the obtained groups. Studied species or breeds are: Abyssinian (ABY), Angora (ANG), Bengali (BEN), Cornish (COR), Maine (MAI), Siamese (SIA), Sphynx (SPH), leopard (LEO), Bobcat (BOB), Eurasian Lynx (EUR) and Iberian Lynx (IBE).

Table 1. Comparison between groups

\begin{tabular}{ccc}
\hline \hline & Wild cats & Domestic cats \\
\hline Domestic cats & $0.026^{*}$ & - \\
Kittens & 0.398 & 0.233 \\
\hline
\end{tabular}

${ }^{*} p<0.05$

yses were performed with the PAST software [8]. A supplementary analysis of similarities (ANOSIM) was performed using also Fourier descriptors as variables.

\section{Results}

Using the Fourier transform, reconstruction of the mean head profile suggested that there was a general shape difference between wild cats, domestic cats and kittens. A visual estimate made from a Fourier series reconstruction using increasing numbers of harmonics is shown in Fig. 2.

The first principal component explained $90.66 \%$ of the total observed variance being very high compared to the second one $(4.46 \%)$, indicating that the first value represented most of the variation. In general, the cumulative contribution ratio of the first three principal components of symmetric variation was $96.85 \%$ of the total dispersal. This result indicated that the profile variation of the head profile was mostly explained by the first principal component.

Specimens plotted in the coordinate system given by the PCO appear in Fig. 3. The ANOSIM indicated a separation between kittens and both wild and domestic cats (Table 1).

\section{Discussion}

Paedomorphic physical traits in domesticated animals (such as dogs, pigs, cats, and recently foxes) include floppy ears, changes in reproductive cycle, curly tails, piebald coloration, fewer or shortened vertebra [20], large eyes, rounded forehead, large ears and shortened muzzle [2].

For the head study, the external outlines (shape analysis through Fourier series) showed its importance. PC1 accounted for more than $90 \%$ of the explained variation and represents the general aspect of the head profile. This clustering might suggest that while the appeareance of superficial juvenile traits occurs in some domestic breeds, such Sphynx and Cornish, and wild felid species, such as bobcats, this appearance is much more minor in others, as Syamese and Leopard. But the similarity between domestic breeds and kittens would be attributed more to the general head profile than to flatness, i.e. to the general conformation rather than to facial profile. Therefore, no effect of profile paedomorphism on the studied domestic breeds can be undertaken.

It could argued that for cats the phenomenon of domestication has been based more on an anthropomorphic than paedomorphic selection because not for all breeds what has been selected is characteristic of juvenile or infantile appearance and behavior. According to Mithen [14], without anthropomorphism, neither pet keeping nor animal domestication would ever have been possible. So, anthropomorphic selection may be the preferable term for the genesis of domestic cats breeds because the putative goal of selection has been to produce animals who are more as breeders like, not always taking into account if their human-like features were also child-like or infantile.

However, further research is needed, including morphometric variables (size and shape, specially referred to physical traits suggestive of paedomorphosis, such size of head, length of legs, and the head-to-body ratio), to complement information. We therefore suggest that future work should aim to collect comparative measures of skull and limb morphology from a range of breeds. In particular, extreme profiled breeds, such as Persian -with a round face and shortened muzzle- and Ukrainian Levkoy -with its dogface appearance- might be included. In addition, we suggest that observations are recorded of the behaviour of single-breed groups during social interactions under standard conditions.

\section{References}

1. Albertson RC, Yan YL, Titus TA, Pisano E, Vacchi M, Yelick PC, Detrichl HW 3rd, Postlethwait JH. Molecular pedomorphism underlies craniofacial skeletal evolution in Antarctic notothenioid fishes. BMC Evol Biol 2010, 10, 4.

2. Bertone J. Equine Geriatric Medicine and Surgery. pp. 288, Saunders, Edinburgh, 2006.

3. Budiansky S. The Nature of Horses: Their Evolution, Intelligence and Behaviour. pp. Phoenix, London, 1998.

4. Freeman H. Computer processing of line-drawing images. ACM Computing Surveys 1974, 6, 57-97.

5. Goodwin D, Bradshaw JWS, Wickens SM. Paedomorphosis affects agonistic visual signals of domestic dogs. Anim Behav 1997, 53, 297-304.

6. Goodwin D, Levine M, McGreevy PD. Preliminary investigation of morphological diffeences between ten breeds of horses suggests selection for paedomorphosis. J App 
Anim Welf Sci 2008, 11, 204-212.

7. Gould SJ. Ontogeny and Phylogeny. pp. 501, Harvard University Press, Cambridge, 1977.

8. Hammer Ø, Harper DAT, Ryan PD. PAST: paleontological statistics software package for education and data analysis. Palaeontol Electronica 2001, 4, 4.

9. Hârupa O. Elliptic Fourier analysis of crown shapes in Quercus petraea trees. Ann For Res 2011, 54, 99-117.

10. Iwata H, Ukai Y. SHAPE: a computer program package for quantitative evaluation of biological shapes based on elliptic Fourier descriptors. J Hered 2002, 93, 384-385.

11. Kuhl FP, Giardina CR. Elliptic Fourier features of a closed contour. Comput Graph Image Process 1982, 18, 236-258.

12. MacFadden B. Fossil horses: Systematics, paleobiology, and evolution of the family Equidae. pp. Cambridge University Press. Cambridge, 1992.

13. Martin DP, Rybicki EP. Microcomputer-based quantification of maize streak virus symptoms in Zea mays. Phytopathology $1998, \mathbf{8 8}, 422-427$.
14. Mithen S. The Prehistory of The Mind: A Search for the Origins of Art, Religion and Science. 1st ed. pp. 288, Thames and Hudson, London, 1996.

15. Morey DF. Size, shape and development in the evolution of the domestic dog. J Archaeol Sci 1992, 19, 181-204.

16. Niemira BA, Kirk WW, Stein JM. Screening for late blight susceptibility in potato tubers by digital analysis of cut tuber surfaces. Plant Dis 1999, 83, 469-473.

17. Olmstead JW, Lang GA. Assessment of severity of powdery mildew infection of sweet cherry leaves by digital image analysis. HortScience 2001, 36, 107-111.

18. Podani J, Miklo I. Resemblance coefficients and the horseshoe effect in principal coordinates analysis. Ecology 2002, 83, 3331-3343.

19. Sheldon P. The evolution of form. In: Skelton PW (ed.). Evolution: A Biological and Paleontological Approach. pp. 723-732, Prentice Hall, Wokinghan, 1993.

20. Trut LN. Early canid domestication: the farm-fox experiment. Am Sci 1999, 87, 160-169. 